\title{
INTERPRETAR O TRABALHO COMO UM CHAMADO CONTRIBUI PARA A EMPREGABILIDADE OU A ATRAPALHA?
}

\author{
http://dx.doi.org/10.21527/2237-6453.2021.55.11031 \\ Recebido em: 26/6/2020 \\ Aceito em: 13/4/2021 \\ Bruno Felix, ${ }^{1}$ Julia Almeida Buaiz Papaleo²
}

\begin{abstract}
RESUMO
Neste estudo sustenta-se que chamados ocupacionais não são nem somente positivos nem somente negativos para a empregabilidade de indivíduos. Para testar esta ideia objetivou-se analisar a existência de associações positivas e negativas indiretas entre a percepção de um chamado ocupacional e a empregabilidade, e o efeito moderador da mentalidade de paradoxo em tais relações. Para tal, foi realizado um estudo de natureza quantitativa e os dados foram analisados por meio da Modelagem de Equações Estruturais. Os resultados mostraram que perceber um chamado ocupacional está associado a uma empregabilidade. Além disso, constatou-se um efeito indireto positivo entre essas variáveis, via desenvolvimento profissional proativo, e um efeito indireto negativo, via inflexibilidade na carreira. Por fim, foi identificado também um efeito moderador da mentalidade de paradoxo na relação entre perceber um chamado e empregabilidade, mediada pela inflexibilidade de carreira. Desse modo, este estudo contribui para a literatura de desenvolvimento de carreiras ao mostrar possíveis efeitos negativos da percepção de um chamado para a empregabilidade, enquanto a prática de muitos aconselhadores de carreira ignoram este efeito.
\end{abstract}

Palavras-chave: Chamado ocupacional. Empregabilidade. Mentalidade de paradoxo. Desenvolvimento profissional proativo.

\section{DOES INTERPRETING WORK AS A CALLING CONTRIBUTE OR DAMAGE AN INDIVIDUAL'S EMPLOYABILITY?}

\section{ABSTRACT}

In this study, we argue that occupational callings are not only positive nor only negative for an individual's employability. For testing this idea, we aimed at analyzing the existence of positive and negative indirect associations between perceiving an occupational calling an employability, and the moderating effect of paradox mindset in those relationships. For such, we conducted a quantitative research and we analyzed our data using Structural Equations Modeling. The results showed a negative direct effect between perceiving an occupational calling and employability. In addition, we found a positive indirect effect between these variables, via proactive professional development, and a negative indirect effect, via career inflexibility. Finally, a moderating effect of the paradox mindset was also identified in the relationship between perceiving a call and employability, mediated by career inflexibility. Thus, this study contributes to the career development literature by showing negative effects of perceiving a calling for employability, while the practice of many career counselors ignore this effect.

Keywords: Occupational calling. Employability. Paradox mindset. Proactive professional development.

\footnotetext{
${ }^{1}$ Autor correspondente. Fucape Business School (FBS). Av. Fernando Ferrari, 1358 - Boa Vista, Vitória/ES, Brasil. CEP $29075-505$. http://lattes.cnpq.br/3237367085252728. https://orcid.org/0000-0001-6183-009X.bfelix@fucape.br

${ }^{2}$ Fucape Business School (FBS). julia.buaiz@gmail.com
} 


\section{INTRODUÇÃO}

No mundo contemporâneo tem crescido a ideia de que o trabalho deve ser não apenas um domínio em que pessoas obtêm seu sustento financeiro, mas também satisfazem um senso existencial mais amplo (DUFFY et al., 2018a). Essa forma de ver o trabalho tem sido teorizada por meio de conceitos como trabalho significativo (ALLAN; AUTIN; DUFFY, 2016), significado do trabalho (DUFFY et al., 2015a) e, mais recentemente, chamados ocupacionais (FELIX; BLULM, 2020), que é uma ocupação a qual um indivíduo se sente inclinado a desempenhar, interpreta como intrinsecamente prazerosa e significativa e vê como parte central de sua identidade (DOBROW; TOSTI-KHARAS, 2010). Em geral, tem sido difundida a ideia de que, ao interpretarem o trabalho como um chamado ocupacional, pessoas potencializam seus resultados em diferentes variáveis relacionadas ao desenvolvimento de suas carreiras (DOBROW; TOSTI-KHARAS, 2011). Entre estas destaca-se a empregabilidade, que é a capacidade do indivíduo de ser escolhido ou instituir uma determinada carreira (HARVEY, 2001; KNIGHT; YORKE, 2002). O raciocínio intuitivo nesse caso é o de que, ao desempenharem um trabalho para o qual se sentem chamados, indivíduos tendem a aumentar suas chances de poder escolher um trabalho a ser desempenhado, uma vez que seriam pessoas mais motivadas para exercício de suas atividades profissionais (LYSOVA et al, 2018).

Pesquisas anteriores, entretanto, mostram que essa associação positiva entre chamados ocupacionais e empregabilidade pode não ser tão óbvia quanto parece em um primeiro momento. Por um lado, estudos entre jovens adultos mostraram que chamados ocupacionais estão associados a uma maior empregabilidade e são responsáveis por uma relação positiva mais forte entre preparação profissional e empregabilidade (PRASKOVA; CREED; HOOD, 2015b, LYSOVA et al., 2018). Por outro, alguns estudiosos também sugeriram que chamados ocupacionais podem prejudicar a obtenção de competências e conhecimentos que estão fora do domínio de chamado (BERKELAAR; BUZZANELL, 2015; NEWPORT, 2012), mas que são necessárias para a empregabilidade (ARTHUR; KHAPOVA; RICHARDSON, 2017).

Diante desses resultados mistos, este estudo adota uma visão de que chamados ocupacionais não são nem somente positivos nem somente negativos para a empregabilidade de indivíduos. Assim, objetivou-se analisar a existência de associações positivas e negativas indiretas entre chamados ocupacionais e a empregabilidade, e o efeito moderador da mentalidade de paradoxo em tais relações. Argumenta-se aqui que a visão de uma carreira como um chamado se relacione positivamente com a empregabilidade via desenvolvimento profissional proativo (HALL; CHANDLER, 2005; VAN DER HEIJDE; VAN DER HEIJDEN, 2006). Por outro lado, sugere-se que a existência de um chamado ocupacional pode levar indivíduos a uma maior inflexibilidade de carreira (LYSOVA et al., 2018), o que pode levar a produzir um efeito negativo indireto na empregabilidade.

Além disto, neste texto também se sustenta a ideia de que as relações positivas entre perceber um chamado ocupacional e as variáveis de desenvolvimento profissional proativo e inflexibilidade de carreira tendem a ser moderadas pela mentalidade de paradoxo. Tal conceito refere-se ao grau em que uma pessoa aceita e é energizada por tensões (MIRON-SPEKTOR et al., 2018). Pessoas com maior mentalidade de paradoxo lidam melhor com pressões simultâneas por demandas contraditórias, assim como pessoas com um chamado as possuem (pressões por trabalho significativo e conseguir viver de seu chamado) (DUFFY et al., 2013). Assim, sugerimos 
que: a) pessoas com alta mentalidade de paradoxo, ao perceberem um chamado, tenderiam a desenvolver um foco proativo maior nas atividades que precisam desenvolver para viver tal chamado e b) tendem a ficar menos inflexíveis. Dessa forma, testamos a tese de que para indivíduos com alta mentalidade de paradoxo os efeitos negativos do chamado estariam menos acentuados, enquanto seus efeitos positivos seriam mais fortes.

Este estudo possui implicações teóricas para a literatura de chamados ocupacionais. Ele permite contestar uma visão romantizada de que chamados ocupacionais seriam uma solução transituacional para a resolução de um amplo problema contemporâneo que assola tantas pessoas: desenvolver uma carreira em um trabalho de significado (DIK; DUFFY, 2009). Nesse processo, tem sido difundida a ideia de que ver o trabalho como um chamado seria uma forma de obter resultados positivos na carreira, dado que "quem ama o que faz tem mais sucesso" (PRASKOVA; CREED; HOOD, 2015a, b). Este estudo contribui para essa literatura ao problematizar o tema, ao sugerir que a escolha por viver um chamado ocupacional pode também apresentar riscos para questões práticas, como a empregabilidade, ao tornar o indivíduo menos flexível a outras possibilidades de carreira.

\section{REFERENCIAL TEÓRICO}

\section{Chamados ocupacionais: o que são?}

Há duas visões mais comuns para se entender o uso do termo "chamado" na literatura sobre carreiras. Tradicionalmente, o termo chamado foi usado na esfera do trabalho para designar ocupações inspiradas por um convite percebido de alguma dimensão superior (frequentemente de um Deus ou do universo) para desempenhar um trabalho com finalidades pró-sociais. Nos últimos anos, no entanto, uma corrente contemporânea de estudos tem utilizado o termo em um sentido diferente. Há hoje o entendimento de que um chamado seja a percepção individual de uma designação advinda de uma fonte externa (por exemplo, Deus, necessidade social, herança familiar) ou interna (motivações pessoais) para desenvolver uma carreira que contribua para um senso de significado/propósito e que é usado para servir aos outros de alguma forma (DUFFY et al., 2018b). Nessa mesma linha, alguns autores afirmam que perceber um chamado ocupacional leva indivíduos a constatarem uma atração significativa pelo trabalho (MICHAELSON; TOSTI-KHARAS, 2019), o que contribui para que eles interpretem o trabalho como parte central de sua identidade (BERG; GRANT; JOHNSON, 2010). Essa corrente contemporânea para o entendimento a respeito do que é um chamado ocupacional é a adotada neste estudo.

De forma geral, há uma tendência a se enxergar chamados ocupacionais como algo que confere a indivíduos um caráter proativo, de bem-estar e bem-sucedido para o trabalho, uma vez que um chamado representa um "convite à ação" que ativa processos de engajamento a metas e realização (DAPALMA; LOPES; ALVES, 2018; LI; YANG, 2018). Não é à toa que na prática empresarial tem havido uma certa "ditadura da felicidade" (BERG; GRANT; JOHNSON, 2010), no sentido de sugerir que indivíduos necessitam desenvolver uma missão de vida alinhada com sua carreira, caso contrário são vistos como profissionais infelizes. A reconhecida empresa de recrutamento e seleção estadunidense chamada Monster.com, por exemplo, utilizou como slogan a expressão "Seu chamado te chama", sugerindo que o trabalho deles seria ajudar indivíduos a se 
(re)colocarem no mercado profissional em profissões vistas como um chamado (MICHAELSON; TOSTI-KHARAS, 2019).

Uma questão, no entanto, tem surgido nos últimos anos e desafiado o status positivo de chamados ocupacionais: Diante de tantas expectativas que são criadas a partir da percepção de um chamado ocupacional, seria plausível sugerir que eles também possam gerar consequências para indivíduos? Estudos recentes sobre o lado sombrio de chamados ocupacionais têm mostrado que sim. Por exemplo, Berg, Grant e Johnson (2010) sugerem que perceber um chamado é algo diferente de vivê-lo e que muitas vezes um chamado percebido não é vivido em razão de dificuldades de desempenho ou outras práticas, como falta de capital de investimento para a qualificação ou dificuldade de colocação profissional. Assim, chamados não atendidos tendem a gerar consequências indesejáveis para indivíduos, como maiores níveis de depressão, ansiedade, sintomas físicos e menores níveis de engajamento no trabalho e de satisfação com a vida (GAZICA; SPECTOR, 2015; NUNES; FELIX, 2019), no entanto, mesmo quando chamados são atendidos, há uma tendência a haver consequências indesejáveis, como estafa mental e síndrome de Burnout (DUFFY et al., 2016; HIRSHI; KELLER; SPURK, 2019).

Neste trabalho sustenta-se a ideia de que chamados são, simultaneamente, fontes de resultados positivos e negativos para a carreira de indivíduos. Analisa-se aqui a relação entre chamados ocupacionais e uma variável pouco explorada nesta literatura: a empregabilidade.

\section{Chamados ocupacionais e empregabilidade}

Empregabilidade refere-se à habilidade de obter e reter um trabalho em uma organização atual ou com um empregador diferente (LYSOVA et al., 2018) e tem se mostrado um importante antecedente de variáveis, como satisfação com a vida e retornos financeiros (VELD; SEMEIJN; VUUREN., 2015). Embora haja relativa concordância a respeito desses entendimentos, duas abordagens centrais se apresentam para o estudo do conceito: baseada em competência e em autopercepção. No primeiro caso, a empregabilidade é vista como um grupo de competências por meio das quais o indivíduo desenvolve uma posição vantajosa no mercado laboral (VAN DER HEIJE; VAN DER HEIJDEN, 2006). No segundo, foca-se na percepção que indivíduos possuem a respeito de sua capacidade de obter e reter um trabalho (VANHERCKE et al., 2014). Como nem sempre as competências adquiridas podem se reverter em uma capacidade de se posicionar no mercado laboral, neste estudo adota-se uma abordagem baseada em autopercepção.

Na literatura existe uma tendência a se propor uma relação positiva direta entre a percepção de um chamado ocupacional e a empregabilidade. Por exemplo, Praskova, Creed e Hood (2015a) identificaram que jovens adultos que possuem um chamado percebido tendem a crer que terão uma melhor empregabilidade futura, uma vez que possuem metas elevadas de desempenho pessoal, assim como alta autoconfiança de que o alcançarão. Resultado similar foi encontrado por Praskova, Creed e Hood (2015b), mas não por Lysova et al. (2018), uma vez que no estudo conduzido por estes últimos não foi encontrada uma relação direta entre os conceitos citados. Apesar dos resultados mistos, pode-se sugerir que a relação tende a ser encontrada, uma vez que chamados geralmente promovem maior autoconsciência e capacidade de adaptação, que são características relevantes para a constituição da empregabilidade (FUGATE; KINICKI; ASHFORTH., 2004). Além disso, chamados têm se mostrado como antecedentes de variáveis como adaptabilidade de carreira (DOUGLASS; DUFFY, 2015) e autoeficácia (KAMINSKY; 
BEHREND, 2015), que podem ser entendidas como construtos que facilitam a formação da empregabilidade (HUANG, 2015; PAJIC et al., 2018). Assim, propõe-se que:

H1: Há uma relação positiva entre perceber um chamado e a empregabilidade.

\section{O efeito indireto positivo via Desenvolvimento Profissional Proativo (DPP)}

Diante de um cenário corporativo de alta competitividade e crescente grau de exigência em termos de desempenho, os trabalhadores precisam, cada vez mais, adotar uma postura de responsabilidade a respeito de suas carreiras (DONALD; BARUCH; ASHLEIGH, 2019). Esse tema tem sido explorado por meio do conceito de Desenvolvimento Profissional Proativo, que pode ser definido como um "engajamento autogerenciado no trabalho e em atividades de aprendizagem e desenvolvimento associados ao trabalho" (LYSOVA et al., 2018, p. 263). Uma vez que chamados ocupacionais tendem a ser acompanhados de um incremento na consciência em relação ao autoconceito (BUIS; FERGUSON; BRISCOE, 2019; LAU; WILKINS-YEL; WONG, 2019), é razoável concluir que tal conhecimento de si possa levar indivíduos que possuem um chamado ocupacional a apresentarem uma postura de maior busca pelo desenvolvimento proativo de suas carreiras. Mais especificamente, a clareza a respeito do autoconceito pode ajudar indivíduos a superarem o gap entre o eu desejado e o eu vivido, posicionando-o com uma postura de maior iniciativa e responsabilidade diante do meio em que se insere (SIRIDETKOON; DEWAELE, 2018). Além disso, possuir um chamado ocupacional tende a levar pessoas a se tornarem mais adaptáveis (AHN; DIK; HORNBACK, 2017), o que também é visto como positivo para o desenvolvimento profissional proativo (TABER; BLANKEMEYER, 2015). Outra evidência relevante de que chamados ocupacionais estejam positivamente associados ao DPP é o achado de que a percepção de um chamado, além de ser um convite percebido a ser, é também uma orientação ao fazer, à ação (ELANGOVAN; PINDER; MCLEAN, M. 2010). Finalmente, chamados já se mostraram positivamente associados ao engajamento ativo no desenvolvimento pessoal (DUFFY et al., 2014) e à proatividade diante de choques negativos de carreira (DUFFY et al., 2015). Assim, sugere-se que:

H2: Há uma relação positiva entre perceber um chamado e o Desenvolvimento Profissional Proativo.

Como há indício de que o Desenvolvimento Profissional Proativo se relaciona positivamente com a Empregabilidade (LYSOVA et al., 2018), sugere-se uma relação positiva indireta entre a percepção de um chamado e a empregabilidade (TYMON; BATISTIC, 2016). Criar uma empregabilidade satisfatória depende de uma contínua aquisição de habilidades e conhecimentos que tornam um indivíduo apto a determinados cargos (NYNLUND; VIROLAINEN, 2018). Isso torna-se ainda mais verdadeiro no instável cenário laboral atual, em que a aprendizagem se apresenta como uma responsabilidade individual e, portanto, mais dependente da proatividade e agência dos sujeitos (THOLEN, 2015). Assim, ao perceber um chamado, faz sentido sugerir que indivíduos se tornem mais inclinados à proatividade (LYSOVA; KHAPOVA, 2019), o que, por sua vez, tende a aumentar as chances de que uma pessoa reforce sua empregabilidade. Desta forma, sugere-se que: 
H3: O Desenvolvimento Profissional Proativo medeia a relação entre perceber um chamado e a empregabilidade.

\section{O efeito indireto negativo via inflexibilidade de carreira}

Se por um lado perceber um chamado parece melhorar a empregabilidade, há também uma outra linha de raciocínio por meio da qual essa relação pode ser negativa. Com o aumento da competitividade no mercado laboral, ser flexível e capaz de abrir mão de determinadas metas em prol de objetivos alternativos mais viáveis tem sido visto como uma característica desejável para a carreira. Caso indivíduos não o façam, tornam-se mais sujeitos a frustrações e estagnação na carreira (BOCCIARDI et al., 2017), o que resulta em maior estresse posterior (FIORI; BOLLMANN; ROSSIER., 2015). Pesquisas anteriores têm sugerido que ver o trabalho como um chamado, no entanto, leva indivíduos a uma menor habilidade de fazer tais adaptações em seus planos. Por exemplo, Dobrow e Tosti-Kharas (2011) sugeriram que um chamado tende a restringir indivíduos a focarem em apenas um caminho para construírem suas carreiras, levando a uma "visão de túnel" (p. 1.002). Por sua vez, destacam que chamados levam indivíduos a uma visão mais estreita a respeito de si, diminuindo sua flexibilidade de carreira, aqui entendida como o grau em que um indivíduo considera caminhos alternativos e se abre a mudanças futuras em suas escolhas de carreira (PORFELI et al., 2011). Assim, sugere-se que:

H4: Há uma relação positiva entre perceber um chamado e a inflexibilidade na carreira.

Propõe-se também aqui que perceber um chamado relaciona-se negativamente com a empregabilidade via inflexibilidade de carreira. Em razão de sua alta inflexibilidade na carreira, pessoas que possuem um chamado podem não ter a habilidade necessária para responder a mudanças em seu segmento laboral ou a desejar fazer ajustes que as levem a maior empregabilidade (LYSOVA; KHAPOVA, 2019). Indivíduos que se sentem chamados a uma ocupação podem estar tão focados em desenvolver expertise para o domínio do seu chamado que falham em desenvolver habilidades generalistas ou em buscar opções alternativas em momentos de escassez de ofertas de trabalho (FELIX; CAVAZOTTE, 2019). Como a disponibilidade para mudar de emprego é um dos antecedentes da empregabilidade (WITTEKIND; RAEDER; GROTE., 2010), é possível que perceber um chamado leve a uma diminuição na empregabilidade por meio de um aumento na inflexibilidade de carreira. Por isso, propõe-se que:

H5: A Inflexibilidade na carreira medeia a relação entre perceber um chamado e a empregabilidade.

\section{O papel moderador da mentalidade de paradoxo}

Perceber um chamado tende a fazer com que indivíduos precisem lidar com uma tensão entre conciliar o atendimento ao chamado com a necessidade de alcançar resultados objetivos na carreira (financeiros, por exemplo) (SCHAMBRAM; MAITILIS, 2017). Pode-se afirmar que a percepção de um indivíduo faz com que os sujeitos vivenciem um paradoxo de carreira. Pessoas geralmente lidam com paradoxos de duas formas principais, ou seja, as que possuem uma elevada mentalidade de dilema costumam lidar com uma tensão de forma a escolher entre uma 
das polaridades (por exemplo, atender ao chamado ou buscar realização financeira) (SMITH; LEWIS, 2011). Por outro lado, as que possuem alta mentalidade de paradoxo tendem a enxergar uma relação de interdependência entre polaridades que apresentam tensão entre si (por exemplo, buscar uma realização financeira por meio do atendimento do chamado e vice-versa).

Apesar da ausência de estudos que tenham avaliado as possíveis relações entre a mentalidade de paradoxo e a percepção de um chamado, é possível teorizar que essa variável potencialize os efeitos positivos indiretos propostos na Hipótese 6 e atenue os efeitos negativos destacados na Hipótese 7. É possível que, ao perceber um chamado, indivíduos não sejam tão proativos na busca por vivê-lo caso acreditem que tal escolha implica renunciar a uma vida financeira próspera (AHN; DIK; HORNBACK, 2017; FELIX; CAVAZOTTE, 2019). Assim, caso haja uma relação de mediação positiva do DPP na relação entre perceber um chamado e a empregabilidade, esse efeito tende a ser mais intenso para indivíduos com menor mentalidade de paradoxo.

Além disso, pode-se sugerir que, ao perceber um chamado, indivíduos tornem-se mais flexíveis no processo de buscar vivê-lo, caso apresentem maior mentalidade de paradoxo. Isso ocorreria uma vez que, para buscar viver um chamado por meio da prosperidade financeira, um indivíduo poderia trabalhar em uma ocupação desprovida de significado por um tempo para financiar a construção de uma carreira associada a um chamado (BERG; GRANT; JOHNSON, 2010). Outra possibilidade seria a de o indivíduo buscar a prosperidade financeira por meio de uma atuação em seu chamado que fosse lucrativa, o que poderia demandar uma maior flexibilidade no processo de definição das atividades ligadas ao chamado (HESLIN, 2005). Assim, caso haja uma relação de mediação negativa da inflexibilidade na relação entre perceber um chamado e a empregabilidade, esse efeito tende a ser menos intenso para indivíduos com menor mentalidade de paradoxo. Desta forma:

H6: O efeito mediador positivo do desenvolvimento profissional proativo na relação entre perceber um chamado e a empregabilidade é mediado pela mentalidade de paradoxo.

Quanto maior a mentalidade de paradoxo, mais forte é tal efeito mediador.

H7: O efeito mediador negativo da inflexibilidade na carreira na relação entre perceber um chamado e a empregabilidade é mediado pela mentalidade de paradoxo.

Quanto maior a mentalidade de paradoxo, mais fraco é tal efeito mediador.

\section{METODOLOGIA}

O objetivo deste estudo é analisar a existência de associações positivas e negativas indiretas entre chamados ocupacionais e a empregabilidade, e o efeito moderador da mentalidade de paradoxo em tais relações. Para tal, foi realizado um estudo de natureza quantitativa, de corte transversal, de forma a testar as hipóteses contidas no modelo teórico apresentado.

A amostra do estudo foi composta por indivíduos que atuassem há pelo menos seis meses no mercado laboral, uma vez que esse público tende a apresentar maior grau de reflexão a respeito de temas como chamados ocupacionais e empregabilidade que indivíduos recém-ingressantes no mercado de trabalho. A coleta de dados ocorreu por meio da disponibilização via e-mail de um questionário on-line para alunos e ex-alunos de MBA de uma instituição de ensino 
na região Sudeste do Brasil. O e-mail foi enviado por um dos autores do estudo. A base é composta por cerca de 4.000 alunos. Como se trata de ex-alunos que possuem vivência profissional, entende-se que tal amostra possibilita o acesso a indivíduos com experiências diversificadas e satisfatória inserção no mercado profissional. No estudo realizado foram mensuradas as seguintes variáveis de controle: gênero, idade, escolaridade, renda, se a pessoa trabalha atualmente e tempo no trabalho atual.

A amostra utilizada no estudo apresentou as seguintes características demográficas: dos 272 respondentes da pesquisa, um total de $62,3 \%$ foi constituído por mulheres e $37,7 \%$ por homens, com uma média de 39 anos de idade, variando entre 19 e 72 anos. 0 grau de escolaridade dos participantes se constituiu da seguinte forma: 0,4\% possuem Ensino Fundamental, 5,2\% estudaram até o Ensino Médio, 24,2\% até o Ensino Superior, 37,3\% são pós-graduados, 27,4\% mestres e 5,6\% estudaram até o Doutorado. A renda mensal dos participantes se configurou da seguinte forma: 3,2\% possuem uma renda mensal de até um salário mínimo, 30,6\% recebem de 1 a 5 salários mínimos, 23,8\% têm uma renda de 5 a 10 salários mínimos, 20,6\% recebem de 10 a 15 salários mínimos e $21,8 \%$ dos respondentes recebem mais do que 15 salários mínimos. Constatou-se que $88,1 \%$ dos entrevistados estão trabalhando, enquanto $11,9 \%$ não desenvolvem uma atividade profissional no momento. Dos que estão trabalhando, $13,4 \%$ estão no atual emprego por um período que varia de 6 meses a 1 ano, 23,4\% trabalham na empresa atual em um período que varia entre 1 e 3 anos, e 63,2\% dos respondentes estão há mais de 4 anos.

Entre as variáveis latentes apresentadas no modelo a ser testado constam as seguintes medidas: perceber um chamado, desenvolvimento profissional proativo, inflexibilidade de carreira, empregabilidade e mentalidade de paradoxo. A seguir apresentam-se as medidas a serem adotadas para cada construto.

a. Chamado Percebido: foi utilizada uma escala de sete itens (Alfa de Cronbach $=0,89$ ) com cinco opções de resposta em uma escala Likert, composta por dois fatores de primeira ordem ("paixão significativa" e "escolha definitiva"). Essa escala é uma adaptação da escala original de Dobrow e Tosti-Kharas (2011), constituída inicialmente para o público de trabalhadores musicistas.

b. Desenvolvimento Profissional Proativo: foi empregada uma escala de três itens, oriunda da escala de cinco itens da dimensão de recursos de trabalho da escala de Job Crafting de Tims, Bakker e Derks (2012). Esses três itens serão usados por capturarem o sentido do construto de Desenvolvimento Profissional Proativo. Tal estratégia foi utilizada anteriormente por Lysova et al. (2018) com um Alfa de Cronbach de 0,88 . As opções de resposta serão numa escala Likert de 5 pontos.

c. Inflexibilidade de carreira: uma escala de 4 itens foi utilizada (Alfa de Cronbach = 0,74), a qual foi construída por Porfeli et al. (2011). As opções de resposta serão dispostas em uma escala Likert de 5 pontos.

d. Empregabilidade: a empregabilidade percebida foi medida por meio da aplicação de uma escala de 3 itens (Alfa de Cronbach $=0,80$ ) de Janssens et al. (2003). As opções de resposta serão dispostas em uma escala Likert de 5 pontos.

e. Mentalidade de paradoxo: foi utilizada uma escala de 9 itens (Alfa de Cronbach = 0,80), proposta por Miron-Spektor et al. (2018). As possibilidades de respostas são apresentadas em uma escala Likert de 5 pontos. 
Para a análise dos dados foi utilizada a metodologia de modelagem de equações estruturais. Inicialmente foi avaliado o modelo de mensuração (validade discriminante, validade convergente e confiabilidade), os índices de ajuste do modelo para, em seguida, apresentar a análise do modelo estrutural (teste das hipóteses).

\section{RESULTADOS E DISCUSSÃO}

\section{Avaliação do modelo de mensuração}

Inicialmente procedeu-se à avaliação das cargas fatoriais dos itens de cada variável latente apresentada no modelo. Todas as variáveis apresentaram cargas fatoriais acima de 0,7 , com exceção da assertiva CP5, que foi excluída do modelo. Os resultados das cargas fatoriais associadas a cada variável latente, assim como as fontes de cada escala, são apresentadas na Tabela 1.

Tabela 1 - Construtos, itens e fontes

\begin{tabular}{|c|c|c|c|}
\hline & \\
\hline & Código & Carga Fatorial & Fonte \\
\hline \multirow{7}{*}{ Chamado Percebido } & CP1 & 0,74 & \multirow{7}{*}{$\begin{array}{l}\text { Adaptado de Dobrow e } \\
\text { Tosti-Kharas (2011) }\end{array}$} \\
\hline & $\mathrm{CP} 2$ & 0,89 & \\
\hline & $\mathrm{CP} 3$ & 0,88 & \\
\hline & CP4 & 0,76 & \\
\hline & CP5 & Excluida & \\
\hline & CP6 & 0,77 & \\
\hline & $\mathrm{CP} 7$ & 0,71 & \\
\hline \multirow{3}{*}{$\begin{array}{c}\text { Desenvolvimento } \\
\text { Profissional Proativo }\end{array}$} & DPP1 & 0,88 & \multirow{3}{*}{$\begin{array}{l}\text { Adaptado de Tims et al. } \\
\qquad(2012) .\end{array}$} \\
\hline & DPP2 & 0,82 & \\
\hline & DPP3 & 0,91 & \\
\hline \multirow{3}{*}{ Empregabilidade } & EMP1 & 0,78 & \multirow{3}{*}{ Janssens et al. (2003) } \\
\hline & EMP2 & 0,84 & \\
\hline & EMP3 & 0,85 & \\
\hline \multirow{4}{*}{ Inflexibilidade de carreira } & IC1 & 0,91 & \multirow{4}{*}{ Porfeli et al. (2011) } \\
\hline & IC2 & 0,92 & \\
\hline & IC3 & 0,88 & \\
\hline & IC4 & 0,79 & \\
\hline \multirow{9}{*}{ Mentalidade de paradoxo } & MP1 & 0,78 & \multirow{9}{*}{$\begin{array}{l}\text { Miron-Spektor et al . } \\
\text { (2018). }\end{array}$} \\
\hline & MP2 & 0,83 & \\
\hline & MP3 & 0,84 & \\
\hline & MP4 & 0,94 & \\
\hline & MP5 & 0,73 & \\
\hline & MP6 & 0,71 & \\
\hline & MP7 & 0,8 & \\
\hline & MP8 & 0,77 & \\
\hline & MP9 & 0,72 & \\
\hline
\end{tabular}

Fonte: Elaboração própria. 
De forma a analisar o modelo de mensuração, foram avaliadas as propriedades de validade convergente, confiabilidade e validade discriminante. Para avaliar a validade convergente observou-se a variância média extraída, e os resultados mostraram valores superiores a 0,5 . Tal resultado sugere a ocorrência da propriedade de validade convergente. Foi encontrada também a presença da propriedade de confiabilidade, uma vez que os valores da confiabilidade composta foram superiores a 0,7 , conforme indicado na Tabela 2 . Nesta, encontram-se também os valores das raízes quadradas das variâncias médias extraídas (valores na diagonal), que, quando superiores aos valores da correlação entre os construtos, indicam a existência de validade discriminante. Conforme é possível observar, a menor das raízes quadradas das variâncias médias extraídas foi de 0,75 , enquanto a maior correlação entre as variáveis latentes foi de 0,43 . esses resultados permitem também inferir a ocorrência de validade discriminante.

Tabela 2 - Avaliação das propriedades psicométricas

\begin{tabular}{lccccc} 
& $\mathbf{1}$ & $\mathbf{2}$ & $\mathbf{3}$ & $\mathbf{4}$ & $\mathbf{5}$ \\
\cline { 2 - 6 } 1 - Chamado Percebido & $\mathbf{0 , 7 8}$ & & & & \\
\hline 2 - Desenvolvimento Profissional Proativo & $-0,21$ & $\mathbf{0 , 8 1}$ & & & \\
\hline 3 - Empregabilidade & $-0,31$ & $-0,03$ & $\mathbf{0 , 7 7}$ & & \\
\hline 4 - inflexibilidade de Carreira & 0,37 & 0,17 & 0,32 & $\mathbf{0 , 7 5}$ & $\mathbf{0 , 7 9}$ \\
\hline 5 - Mentalidade de Paradoxo & 0,09 & 0,43 & $-0,14$ & $-0,18$ & \\
\hline & & & & & \\
\hline Variância Média Extraída & 0,61 & 0,66 & 0,59 & 0,56 & 0,62 \\
\hline Confiabilidade Composta & 0,83 & 0,88 & 0,82 & 0,81 & 0,91 \\
\hline
\end{tabular}

Nota: Na diagonal encontram-se a raiz quadrada das variâncias médias extraídas, uma vez que quando esses valores são superiores às correlações entre os construtos (valores fora da diagonal) nota-se a existência de validade discriminante.

Fonte: Elaboração própria (2020).

\section{Avaliação dos índices de ajuste do modelo}

Após a validação do modelo de mensuração, procedeu-se à avaliação da adequação do modelo estrutural, que foi realizada por meio da avaliação da adequação dos índices de ajuste do modelo, os quais são apresentados na Tabela 3. A adequação do modelo estrutural foi avaliada em dois passos: inicialmente apenas com as variáveis de controle, e em seguida com o modelo completo, incluindo as variáveis latentes apresentadas nas hipóteses. Conforme sugerido por Hair et al. (2006), para modelos testados em amostras superiores a 250 observações e um número de variáveis observadas entre 12 e 30, os índices que sugerem um modelo satisfatório são: $\chi^{2} / \mathrm{df}$ (qui-quadrado por graus de liberdade), precisam ser iguais ou inferiores a 3.0; RMSEA (root mean square error of approximation), devem ser inferiores a 0.08 , CFI (comparative fit index) deve superar 0.92 e o SRMR deve ser inferior a 0.08. Como se nota na Tabela 3, todos esses critérios foram cumpridos, o que permite constar índices satisfatórios de ajuste. 
Tabela 3 - Índices de ajuste do modelo

\begin{tabular}{lcccc}
\hline Modelo & $\chi^{2} /$ df & CFI & RMSEA (1.C. - 90\%) & SRMR \\
\hline Mensuração - AFC & 2,43 & 0,91 & $0,069(0,061$ a 0,076) & 0,075 \\
\hline Estrutural - Controles & 2,22 & 0,97 & $0,064(0,045$ a 0,082) & 0,045 \\
\hline Estrutural - Modelo Completo & 2,31 & 0,92 & $0,064(0,055$ a 0,071) & 0,08 \\
\hline
\end{tabular}

Fonte: Elaboração própria (2020).

Nota: Legenda: AFC: análise fatorial confirmatória; Modelo 0: modelo estrutural com as variáveis de controle (sexo, idade, escolaridade, renda, existência de atividade de profissional atual, tempo no trabalho atual); Modelo Completo: Modelo que inclui todas as variáveis latentes e os efeitos moderadores.

\section{Teste das hipóteses (avaliação do modelo de mensuração) e discussão}

A última etapa da análise dos dados foi a avaliação do modelo de mensuração, na qual se testaram as hipóteses apresentadas. Na Figura 1 apresentam-se os resultados do teste realizado.

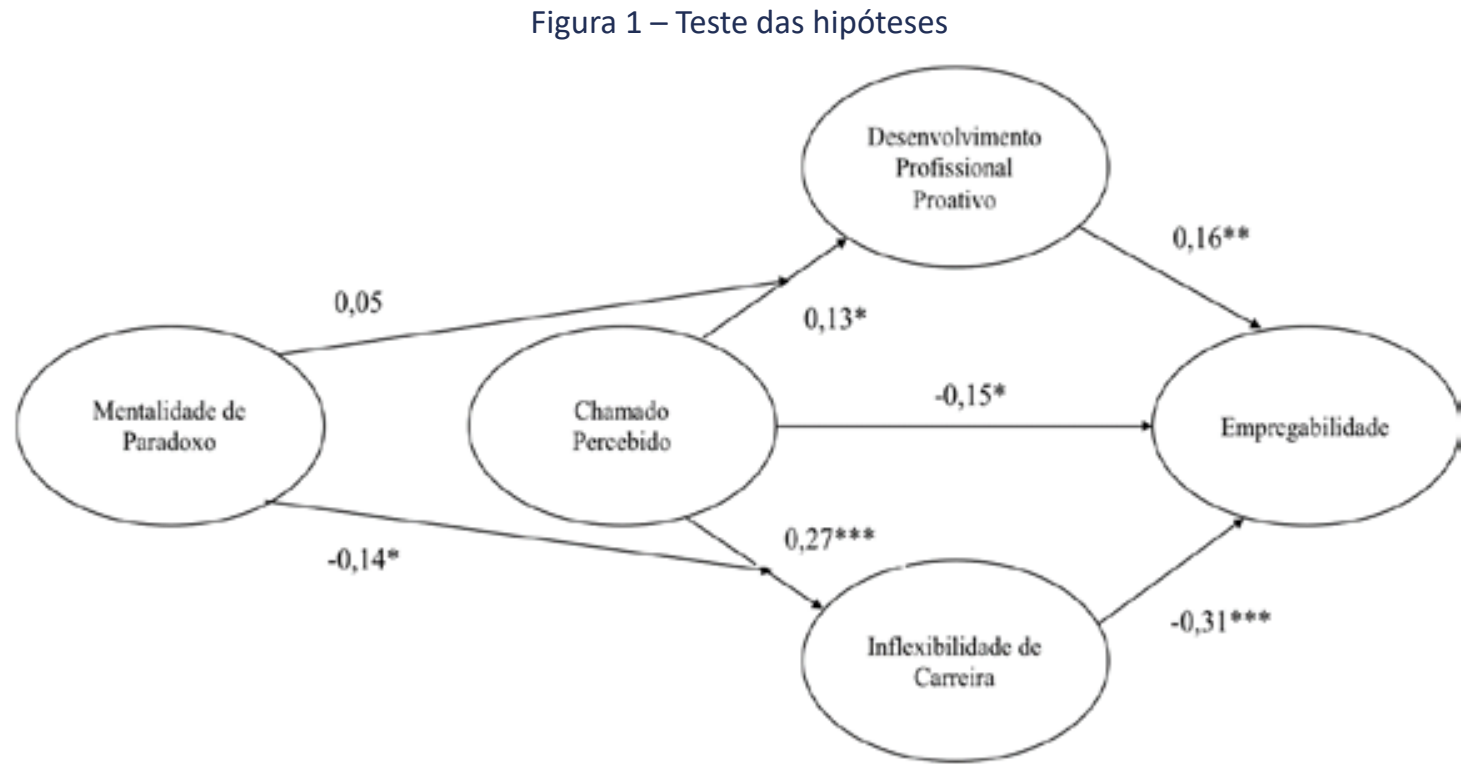

${ }^{*} p<0,05, * * p<0,01, * * * p<0,001$

Fonte: Elaboração própria (2020).

A Hipótese 1, que avaliou a relação positiva entre perceber um chamado e a empregabilidade, foi rejeitada (Coeficiente de caminhos $=-0,15 ; p<0,05$ ). Diferentemente do que foi proposto, o resultado mostrou que o efeito direto do chamado percebido na empregabilidade foi negativo e significante, revelando que ver o trabalho como um chamado tende a diminuir a empregabilidade de indivíduos. Tal resultado conflita como os encontrados no estudo de Praskova, Creed e Hood. (2015a), em que ambas as variáveis apresentaram uma relação positiva. É possível que o fato de o presente estudo ser realizado em um país de economia em desenvolvimento, enquanto o de Praskova, Creed e Hood (2015a) ter sido realizado em um país desenvolvido 
economicamente, explique essa diferença. No entanto, a rejeição de $\mathrm{H} 1$ pode ser mais bem compreendida mediante a análise dos resultados das demais hipóteses.

As Hipóteses 2 e 3, que avaliaram a influência do Desenvolvimento Profissional Proativo na relação com a empregabilidade, não foram rejeitadas. De fato, o chamado percebido relacionou-se com o Desenvolvimento Profissional Proativo, que, por sua vez, gerou um efeito positivo à empregabilidade. A Hipótese 2 foi apoiada por um coeficiente de caminhos de $0,13(p<0,05)$, evidenciando que existe uma relação positiva entre o chamado percebido e o DPP. Esse resultado alinha-se com o encontrado por Elangovan, Pinder e MClean . (2010), os quais evidenciaram que chamados ocupacionais se associaram positivamente ao DPP. Assim, encontra-se uma robustez maior para a ideia de que percepção de um chamado, além de ser uma atitude relativa ao trabalho, caracteriza-se também como uma orientação ao fazer, à ação. Os resultados da Hipótese 3, que sugeriu que o Desenvolvimento Profissional Proativo medeia a relação entre perceber um chamado e a empregabilidade, foram apoiados por coeficientes de $0,13(p<0,05)$ e $0,16(p<0,01)$ e também não foi rejeitada. Esse resultado reforça os achados de Lysova e Khapova (2019), que concluíram que, ao perceber um chamado, indivíduos tornam-se mais inclinados à proatividade, e isso, por sua vez, tende a aumentar as chances de que uma pessoa aumente sua empregabilidade.

Já as Hipóteses 4 e 5 avaliaram o efeito indireto negativo da percepção do chamado na empregabilidade via inflexibilidade de carreira. A Hipótese 4 analisou a existência de uma relação positiva entre perceber um chamado e a inflexibilidade na carreira, e tal relação foi confirmada com um coeficiente de $0,27(p<0,001)$, o que levou à não rejeição dessa hipótese. Tal resultado alinha-se ao proposto por Nunes e Felix (2019), que destacam que chamados levam indivíduos a uma visão mais estreita a respeito de si, diminuindo sua flexibilidade de carreira. Assim como Wittekind, Raeder e Grote (2010), os quais mostraram em seu estudo como a disponibilidade para mudar de emprego é um dos antecedentes da empregabilidade, é possível que perceber um chamado levou a uma diminuição na empregabilidade por meio de um aumento na inflexibilidade de carreira, o que foi evidenciado por meio da não rejeição da Hipótese 5. Foram encontrados coeficientes de caminho de $0,27(p<0,001)$ e $-0,31(p<0,1)$ para esse caminho da mediação.

O chamado percebido aumentando a inflexibilidade de carreira, que por sua vez diminui a empregabilidade, transforma a relação em uma faca de dois gumes, sendo Hipótese 2 o lado positivo e o outro lado negativo, Hipótese 4, o chamado percebido aumentando a inflexibilidade de carreira e diminuindo a empregabilidade. $\mathrm{E}$ com isso nota-se que os efeitos indiretos do chamado na empregabilidade, via inflexibilidade de carreira, foram mais fortes do que os efeitos diretos do chamado percebido na empregabilidade. Isso pode ser uma explicação para a identificação de um efeito direto negativo da percepção do chamado na empregabilidade, em oposição ao encontrado por Duffy et al. (2016) e Praskova, Creed e Hood (2015a).

As Hipóteses 6 e 7 avaliaram o papel moderador da mentalidade de paradoxo nos efeitos mediadores positivo e negativo apresentados nas Hipóteses 3 e 5. Na Hipótese $\mathrm{H} 6$ o efeito mediador positivo do Desenvolvimento Profissional Proativo na relação entre perceber um chamado e a empregabilidade não foi moderado pela mentalidade de paradoxo (coeficiente de caminho $=0,05$, n.s.). Por sua vez, a Hipótese 7 foi não foi rejeitada, dado que foi identificado um coeficiente de caminho de $-0,14(p<0,05)$. Isso permite afirmar que quanto mais mentalidade de paradoxo a pessoa tem, menor será o efeito indireto negativo da percepção de um chamado 
na empregabilidade, via inflexibilidade na carreira. Em outras palavras, para as pessoas com alta mentalidade de paradoxo (SMITH; LEWIS, 2011; MIRON-SPEKTOR et al., 2018), o efeito indireto negativo do chamado na empregabilidade é atenuado.

A escolha por viver um chamado percebido, juntamente com os resultados obtidos neste estudo, apresenta algumas contribuições específicas relevantes para esse campo de pesquisa sobre interpretar um chamado contribuir ou atrapalhar a empregabilidade, tais como que a escolha por viver um chamado ocupacional pode também apresentar riscos para questões práticas, como a empregabilidade (NYLUND; VIROLAINEN, 2018), ao tornar o indivíduo menos flexível a outras possibilidades de carreira; contestar uma visão romantizada de que chamados ocupacionais seriam uma solução transituacional para desenvolver uma carreira em um trabaIho de significado (FELIX; BLULM, 2020).

\section{CONCLUSÃO E CONSIDERAÇÕES FINAIS}

A principal conclusão à qual se chega a partir do presente estudo refere-se ao fato de que, no contexto de realização desta pesquisa, perceber o trabalho como um chamado levou a efeitos mais positivos que negativos para a empregabilidade, diferentemente do que tem sido amplamente disseminado por orientadores de carreira. Esse resultado mostra a importância de que se realizem pesquisas sobre chamados ocupacionais no contexto de países em desenvolvimento, dado que os achados frequentemente encontrados em países desenvolvidos podem não ser encontrados em outros cenários de maior escassez de oportunidades. Além disso, o estudo também permite mostrar que, para pessoas com maior mentalidade de paradoxo, a percepção do chamado tende a conduzir indivíduos a uma atenuação no efeito negativo indireto da percepção chamado na empregabilidade.

Os resultados desta pesquisa trazem contribuições teóricas relevantes, contribuindo com a literatura sobre o chamado. Os resultados avançam na pesquisa sobre como chamados ocupacionais impactam resultados de carreira (DUFFY; DIK, 2013). Em particular, enriquece-se aqui a compreensão de como e por meio de quais mecanismos o chamado se relacionou negativamente à empregabilidade em um contexto de um país em desenvolvimento. Mostramos que os efeitos indiretos do chamado na empregabilidade, via inflexibilidade de carreira, foram mais fortes do que os efeitos diretos do chamado percebido na empregabilidade. Esse resultado permite ampliar o entendimento a respeito dos lados sombrios de se ver o trabalho como um chamado, o que vem sendo realizado em diversos estudos (DUFFY et al., 2016; DUFFY; DIK, 2013).

Este estudo possui limitações e oferece também oportunidades para futuras pesquisas. Primeiro, foram coletados dados em apenas um país em desenvolvimento, o que não permite afirmar que as diferenças nos achados na relação entre a percepção do chamado e a empregabilidade efetivamente se deva às condições do mercado do país em que a pesquisa foi realizada. Assim, seria interessante que um estudo futuro buscasse testar tal associação em outros países em desenvolvimento. Segundo, o estudo analisou apenas um mecanismo de atenuação da relação negativa indireta entre perceber um chamado e empregabilidade: a mentalidade de paradoxo. Um estudo posterior poderá testar o efeito de outras variáveis moderadoras, como a volição do trabalho (DUFFY et al., 2015b) e o acesso a oportunidades (DUFFY et al., 2017). Isso possibilitaria um melhor entendimento acerca de como os efeitos negativos aqui discutidos podem ser amenizados. 
Em termos práticos, o estudo também apresenta implicações relevantes para orientadores de carreira e profissionais. Orientadores de carreira podem se beneficiar ao desenvolverem um melhor entendimento a respeito das possíveis consequências indesejadas que a prática recorrente de se estimular que indivíduos vejam o trabalho como um chamado pode gerar para a empregabilidade desses sujeitos. O caráter intuitivo que leva indivíduos a acreditarem que "quem faz o que ama" necessariamente terá maiores possibilidades em termos de carreira foi contestado pelas evidências do presente estudo. Assim, indivíduos que desenvolvem suas carreiras no contexto aqui estudado são estimulados a reverem a crença absoluta, caso haja uma percepção de que seus chamados ocupacionais necessariamente os levarão a uma maior capacidade de se posicionar profissionalmente. Aqueles, no entanto, que já possuem a percepção de um chamado ocupacional podem atenuar os possíveis efeitos negativos deste em sua empregabilidade ao buscar conciliar os paradoxos inerentes ao chamado percebido (por exemplo, conciliando o idealismo de um chamado com a necessidade de flexibilizar atividades a serem desempenhadas de forma a aumentarem a chance de obter ganhos financeiros).

\section{REFERÊNCIAS}

AHN, Jina; DIK, Bryan J.; HORNBACK, Ruby. The experience of career change driven by a sense of calling: An interpretative phenomenological analysis approach. Journal of Vocational Behavior, v. 102, p. 48-62, 2017.

ALLAN, Blake A.; AUTIN, Kelsey L.; DUFFY, Ryan D. Self-determination and meaningful work: Exploring socioeconomic constraints. Frontiers in Psychology, v. 7, p. 71, 2016.

ARTHUR, Michael B.; KHAPOVA, Svetlana N.; RICHARDSON, Julia. An Intelligent Career: Taking ownership of your work and your life. Oxford: Oxford University Press, 2017.

BERG, Justin M.; GRANT, Adam M.; JOHNSON, Victoria. When callings are calling: Crafting work and leisure in pursuit of unanswered occupational callings. Organization Science, v. 21, n. 5, p. 973-994, 2010.

BERKELAAR, Brenda L.; BUZZANELL, Patrice M. Bait and switch or double-edged sword? The (sometimes) failed promises of calling. Human relations, v. 68, n. 1, p. 157-178, 2015.

BOCCIARDI, Federica et al. Career adaptability as a strategic competence for career development. European Journal of Training and Development, v. 41, n. 1, p. 67-82, 2017.

BUIS, Brittany C.; FERGUSON, Amanda J.; BRISCOE, Jon P. Finding the "I" in "Team": The role of groups in an individual's pursuit of calling. Journal of Vocational Behavior, v. 114, p. 88-99, 2019.

BUNDERSON, J. Stuart; THOMPSON, Jeffery A. The call of the wild: Zookeepers, callings, and the double-edged sword of deeply meaningful work. Administrative science quarterly, v. 54, n. 1, p. 32-57, 2009.

DA PALMA, Patricia Jardim; LOPES, Miguel Pereira; ALVES, Telmo Ferreira. Entrepreneurship as a Calling: A Pilot Study with Aspiring Entrepreneurs. The Journal of Entrepreneurship, v. 27, n. 2, p. 277-300, 2018.

DIK, Bryan J. et al. Development and validation of the calling and vocation questionnaire (CVQ) and brief calling scale (BCS). Journal of Career Assessment, v. 20, n. 3, p. 242-263, 2012.

DIK, Bryan J.; DUFFY, Ryan D. Calling and vocation at work: Definitions and prospects for research and practice. The counseling psychologist, v. 37, n. 3, p. 424-450, 2009.

DOBROW, S. R.; TOSTI-KHARAS, J. Calling a "calling" a calling: The development of a scale measure. Personnel Psychology, v. 64, p. 23-45, 2010.

DOBROW, S. R.; TOSTI-KHARAS, Jennifer. Calling: The development of a scale measure. Personnel psychology, v. 64, n. 4, p. 1.001-1.049, 2011.

DONALD, William E.; BARUCH, Yehuda; ASHLEIGH, Melanie. The undergraduate self-perception of employability: human capital, careers advice, and career ownership. Studies in Higher Education, v. 44, n. 4, p. 599-614, 2019.

DOUGLASS, Richard P.; DUFFY, Ryan D. Calling and career adaptability among undergraduate students. Journal of Vocational Behavior, v. 86, p. 58-65, 2015.

DUFFY, R. D. et al. Calling among the unemployed: Examining prevalence and links to coping with job loss. The Journal of Positive Psychology, v. 10, n. 4, p. 332-345, 2015a. 
DUFFY, R. D. et al. Work as a calling: A theoretical model. Journal of Counseling Psychology, v. 65, n. 4, p. 423, 2018a.

DUFFY, R. D. et al. Calling and life satisfaction: It's not about having it, it's about living it. Journal of Counseling Psychology, v. 60, n. 1, p. 42, 2013.

DUFFY, R. D.; AUTIN, Kelsey L.; BOTT, Elizabeth M. Work volition and job satisfaction: Examining the role of work meaning and person - environment fit. The Career Development Quarterly, v. 63, n. 2, p. 126-140, 2015.

DUFFY, R. D. et al. Calling among the unemployed: examining prevalence and links to coping with job loss. The Journal of Positive Psychology, v. 10, n. 4, p. 332-345, 2015b.

DUFFY, R. D. et al. Perceiving a calling and well-being: Motivation and access to opportunity as moderators. Journal of Vocational Behavior, v. 98, p. 27-137, 2017.

DUFFY, R. D. et al. Work as a calling: A theoretical model. Journal of Counseling Psychology, v. 65, n. 4, p. 423-435, 2018b.

DUFFY, R. D.; DIK, Bryan J. Research on calling: What have we learned and where are we going? Journal of Vocational Behavior, v. 83, n. 3, p. 428-436, 2013.

DUFFY, R. D. et al. Examining predictors and outcomes of a career calling among undergraduate students. Journal of Vocational Behavior, v. 85, n. 3, p. 309-318, 2014.

DUFFY, R. D. et al. Does the dark side of a calling exist? Examining potential negative effects. The journal of positive psychology, v. 11, n. 6, p. 634-646, 2016.

ELANGOVAN, Anbalagan R.; PINDER, Craig C.; MCLEAN, Murdith. Callings and organizational behavior. Journal of Vocational Behavior, v. 76, n. 3, p. 428-440, 2010.

FELIX, Bruno; BLULM, Luiz Felipe Magnago. Comparando os impactos da percepção do chamado ocupacional na vida pessoal, profissional e na saúde dos funcionários públicos. Desenvolvimento em Questão, Ijuí: Editora Unijuí, v. 18, n. 51, p. 281-296, 2020.

FELIX, Bruno; CAVAZOTTE, Flavia. When a Calling Goes Unanswered: Exploring the Role of Workplace Personalizations as Calling Enactments. Frontiers in psychology, v. 10, 2019.

FIORI, Marina; BOLLMANN, Grégoire; ROSSIER, Jérôme. Exploring the path through which career adaptability increases job satisfaction and lowers job stress: The role of affect. Journal of Vocational Behavior, v. 91, p. 113-121, 2015.

FUGATE, Mel; KINICKI, Angelo J.; ASHFORTH, Blake E. Employability: A psycho-social construct, its dimensions, and applications. Journal of Vocational behavior, v. 65, n. 1, p. 14-38, 2004.

GAZICA, Michele W.; SPECTOR, Paul E. A comparison of individuals with unanswered callings to those with no calling at all. Journal of Vocational behavior, v. 91, p. 1-10, 2015.

HAIR, Joseph et al. Multivariant Data Analysis. New Jersey: Pearson International Edition, 2006.

HALL, Douglas T.; CHANDLER, Dawn E. Psychological success: When the career is a calling. Journal of Organizational Behavior, v. 26, n. 2, p. 155-176, 2005.

HARVEY, Lee. Defining and measuring employability. Quality in higher education, v. 7, n. 2, p. 97-109, 2001.

HESLIN, Peter A. Conceptualizing and evaluating career success. Journal of Organizational Behavior: The International Journal of Industrial, Occupational and Organizational Psychology and Behavior, v. 26, n. 2, p. 113-136, 2005.

HIRSCHI, Andreas; HERRMANN, Anne. Calling and career preparation: Investigating developmental patterns and temporal precedence. Journal of Vocational Behavior, v. 83, n. 1, p. 51-60, 2013.

HIRSCHI, Andreas; KELLER, Anita C.; SPURK, Daniel. Calling as a double-edged sword for work-nonwork enrichment and conflict among older workers. Journal of Vocational Behavior, v. 114, p. 100-111, 2019.

HUANG, Jie-Tsuen. Hardiness, perceived employability, and career decision self-efficacy among Taiwanese college students. Journal of Career Development, v. 42, n. 4, p. 311-324, 2015.

KAMINSKY, Samuel E.; BEHREND, Tara S. Career choice and calling: Integrating calling and social cognitive career theory. Journal of Career Assessment, v. 23, n. 3, p. 383-398, 2015.

KNIGHT, Peter T.; YORKE, Mantz. Employability through the curriculum. Tertiary education and management, v. 8, n. 4, p. 261-276, 2002.

JANSSENS, M.; SELS, L.; VAN DEN BRANDE, I. Multiple types of psychological contracts: A six-cluster solution. Human Relations; Studies Towards the Integration of the Social Sciences, v. 56, n. 11, p. 1.3491.378, 2003. 
LAU, Poh Li; WILKINS-YEL, Kerrie G.; WONG, Y. Joel. Examining the Indirect Effects of Self-Concept on Work Readiness Through Resilience and Career Calling. Journal of Career Development, v. 47, n. 5, p. 551-564, 2019. Doi: 0894845319847288

LI, Hongxia; YANG, Xiugang. When a calling is living: Job crafting mediates the relationships between living a calling and work engagement. Journal of Chinese Human Resource Management, 2018.

LYSOVA, Evgenia I. et al. Examining calling as a double-edged sword for employability. Journal of Vocational Behavior, v. 104, p. 261-272, 2018.

LYSOVA, Evgenia I.; KHAPOVA, Svetlana N. Enacting creative calling when established career structures are not in place: the case of the Dutch video game industry. Journal of Vocational Behavior, v. 114, p. 31-43, 2019.

MICHAELSON, Christopher; TOSTI-KHARAS, Jennifer. Serving self or serving others? Close relations' perspectives on ethics and calling. Journal of Vocational Behavior, v. 114, p. 19-30, 2019.

MIRON-SPEKTOR, Ella et al. Microfoundations of organizational paradox: The problem is how we think about the problem. Academy of Management Journal, v. 61, n. 1, p. 26-45, 2018.

NEWPORT, C. So good they can't ignore you: why skills trump passion in the quest for work you love. New York: Business Plus, 2012.

NUNES, Fernando Antonio Tocantins; FELIX, Bruno. Viver um chamado ocupacional por meio do empreendedorismo leva à satisfação no trabalho? Revista Pensamento Contemporâneo em Administração, v. 13, n. 4, p. 100-115, 2019.

NYLUND, Mattias; VIROLAINEN, Maarit. Balancing "flexibility" and "employability": The changing role of general studies in the Finnish and Swedish VET curricula of the 1990s and 2010s. European Educational Research Journal, v. 18, n. 3, p. 314-334, 2018.

PAJIC, Sofija et al. Antecedents and outcomes of Hungarian nurses' career adaptability. International Journal of Manpower, v. 39, n. (8), p. 1.096-1.114, 2018.

PORFELI, Erik J. et al. A multi-dimensional measure of vocational identity status. Journal of adolescence, v. 34, n. 5, p. 853-871, 2011.

PRASKOVA, Anna; CREED, Peter A.; HOOD, Michelle. Career identity and the complex mediating relationships between career preparatory actions and career progress markers. Journal of Vocational Behavior, v. 87, p. 145-153, 2015b.

PRASKOVA, Anna; CREED, Peter A.; HOOD, Michelle. Self-regulatory processes mediating between career calling and perceived employability and life satisfaction in emerging adults. Journal of Career Development, v. 42, n. 2, p. 86-101, 2015a.

SCHABRAM, Kira; MAITLIS, Sally. Negotiating the challenges of a calling: Emotion and enacted sensemaking in animal shelter work. Academy of Management Journal, v. 60, n. 2, p. 584-609, 2017.

SIRIDETKOON, Pitchayapa; DEWAELE, Jean-Marc. Ideal self and ought-to self of simultaneous learners of multiple foreign languages. International Journal of Multilingualism, v. 15, n. 4, p. 313-328, 2018.

SMITH, Wendy K.; LEWIS, Marianne W. Toward a theory of paradox: A dynamic equilibrium model of organizing. Academy of management Review, v. 36, n. 2, p. 381-403, 2011.

TABER, Brian J.; BLANKEMEYER, Maureen. Future work self and career adaptability in the prediction of proactive career behaviors. Journal of Vocational Behavior, v. 86, p. 20-27, 2015.

THOLEN, Gerbrand. What can research into graduate employability tell us about agency and structure? British Journal of Sociology of Education, v. 36, n. 5, p. 766-784, 2015.

TIMS, Maria; BAKKER, Arnold B.; DERKS, Daantje. Development and validation of the job crafting scale. Journal of vocational behavior, v. 80, n. 1, p. 173-186, 2012.

TYMON, Alex; BATISTIC, Sasa. Improved academic performance and enhanced employability? The potential double benefit of proactivity for business graduates. Teaching in Higher Education, v. 21, n. 8, p. 915932, 2016.

VAN DER HEIJDE, Claudia M.; VAN DER HEIJDEN, Beatrice I. J. M. A competence-based and multidimensional operationalization and measurement of employability. Society of Human Resources Management, v. 45, n. 3, p. 449-476, 2006.

VAN DER HEIJDEN, Beatrice. Prerequisites to guarantee life-long employability. Personnel Review, v. 31, n. 1, p. 44-61, 2001.

VANHERCKE, Dorien et al. Defining perceived employability: a psychological approach. Personnel Review, v. 43, p. 592-605, 2014. 
INTERPRETAR O TRABALHO COMO UM CHAMADO CONTRIBUI PARA A EMPREGABILIDADE OU A ATRAPALHA? Bruno Felix - Julia Almeida Buaiz Papaleo

VELD, Monique; SEMEIJN, Judith; VAN VUUREN, Tinka. Enhancing perceived employability. Personnel Review, v. 44, n. 6, p. 866-882, 2015.

WITTEKIND, Anette; RAEDER, Sabine; GROTE, Gudela. A longitudinal study of determinants of perceived employability. Journal of Organizational Behavior, v. 31, n. 4, p. 566-586, 2010. 